\title{
Dynamic mechanical behavior of starch-based scaffolds in dry and physiologically simulated conditions: Effect of porosity and pore size
}

\author{
Satyabrata Ghosh ${ }^{\mathrm{a}, \mathrm{b}}$, Victor Gutierrez ${ }^{\mathrm{a}, \mathrm{b}}$, Carolina Fernández ${ }^{\mathrm{a}, \mathrm{b}}$, \\ Miguel A. Rodriguez-Perez ${ }^{c}$, Júlio C. Viana ${ }^{\mathrm{d}}$, Rui L. Reis ${ }^{\mathrm{a}, \mathrm{b}}$, João F. Mano ${ }^{\mathrm{a}, \mathrm{b}, *}$ \\ a 3 Bs Research Group (Biomaterials, Biodegradables and Biomimetics), Department of Polymer Engineering, University of Minho, Braga 4710-057, Portugal \\ ${ }^{\mathrm{b}}$ Institute for Biotechnology and Bioengineering (IBB), PT Government Associated Laboratory, Braga, Portugal \\ ${ }^{\mathrm{c}}$ Department of Condensed Matter Physics, University of Valladolid, E-47011, Valladolid, Spain \\ ${ }^{\mathrm{d}}$ Institute for Polymers and Composites (IPC), Department of Polymer Engineering, University of Minho, Guimarães 4800-058, Portugal
}

Received 27 October 2007; received in revised form 28 January 2008; accepted 4 February 2008

Available online 15 February 2008

\begin{abstract}
The three-dimensional scaffolds of a blend of starch and poly(L-lactic) acid, SPLA70, were produced using compression molding of polymer/salt mixture followed by leaching of salt. One series of scaffolds were prepared with varying polymer-to-salt ratio while keeping the salt size constant, and the other series of scaffolds were prepared with varying salt sizes while keeping the polymer-to-salt ratio constant. The X-ray microcomputed tomography and scanning electron microscopy assay were used to analyze the porous morphologies, porosity and distribution of porosity of the porous scaffolds. Salt-free and integrated SPLA70 scaffolds with porosities ranging from $74 \%$ to $82 \%$ and pore sizes of $125-250$ to $500-1000 \mu \mathrm{m}$ can be fabricated using the present fabrication technique. The water uptake of the SPLA70 scaffolds increases with increasing porosities and also with increasing pore size. In dry state, the storage modulus decreases with increasing porosity and also with increasing pore size. The normalized modulus values are related to normalized density of the scaffolds by a power-law function with an exponent between 2 and 3. For the immersed scaffolds under physiological conditions, the storage modulus was less dependent on porosity and pore size. However, the loss factor increased significantly compared with dry state measurements. The present study clearly shows that the mechanical performance of porous polymeric constructs in dry and in immersed state is completely different, and for comparison with biomechanical performance of tissues, the tests should ideally be performed in immersed state.
\end{abstract}

(C) 2008 Acta Materialia Inc. Published by Elsevier Ltd. All rights reserved.

Keywords: Dynamic mechanical properties; Porosity; Pore size; Dry and immersed state; Starch blend

\section{Introduction}

Tissue engineering approaches have great potential in the biological and functional regeneration of tissues, allowing for overcoming the lack of donor tissue and to promote a biologically and mechanically functional tissue. An

\footnotetext{
${ }^{*}$ Corresponding author. Address: 3Bs Research Group (Biomaterials, Biodegradables and Biomimetics), Department of Polymer Engineering, University of Minho, Braga 4710-057, Portugal. Tel.: +351 253604497.

E-mail address: jmano@dep.uminho.pt (J.F. Mano).
}

important step to engineer tissues is the development of porous three-dimensional scaffolds for different anatomical locations in the body. Generally, the materials of scaffolds are either of natural origin or synthetic biodegradable polymers [1-4]. Synthetic polymers have design flexibilities in terms of materials composition, processability, control over macro- and microstructures, and mechanical properties [4]. These properties can be tuned for specific applications. However, natural polymers are also used in biomedical applications for their excellent biocompatibility and biodegradability $[3,5]$. 
Poly( $\alpha$-hydroxy acids) including poly(lactic acid) (PLA), poly(glycolic acid) (PGA) and their co-polymers (PLGA) are the widely accepted polymers for most of the tissue engineering applications [6]. These thermoplastic polyesters have reasonable biocompatibility, biodegradability, mechanical strength and easy processability. Blends of PLLA and starch (SPLA) have also been proposed in our group to be used in the biomedical field, and were found from cell adhesion and proliferation tests, to interact positively with cells [7].

The fabrication of porous scaffolds from a polymer melt allows fast production of scaffolds with different shape and sizes in an efficient way. The most straightforward way is to melt a polymer of a polymer/salt mixture, cool it and subsequently remove the salt particles, thus producing the porous scaffolds. The desired porosity and pore sizes could be independently controlled by the fractions and sizes of salt particles, respectively $[8,9]$.

The mismatch between the mechanical properties of scaffolds and the tissue intended to be regenerated may compromise the clinical success of the tissue engineering construct. In this regard, stiffness is a relevant mechanical property of scaffolds as it will determine the deformability of the structure upon implantation. The modulus of elasticity describes the slope of the stress-strain relationship for a given material under load. Such property depends strongly on the porosity in the case of a porous system, and may also be influenced by pore size [10]. In the case of polymeric-based materials, one should also consider their viscoelastic properties as they will determine the capability of the device to dissipate mechanical energy and are responsible for the time-dependent nature of its mechanical performance. Moreover, living tissue also exhibits a clear viscoelastic behavior and, ideally, the implantable scaffold should present similar properties in order to maintain the contact with the surrounding tissue during all the physiological stress-strain history. Of course, this would be difficult to achieve using polymer-based materials intended to be used in the replacement or regeneration of hard tissues. Although some rheological properties could be similar in biomaterial and tissue (e.g. damping), stiffness-related properties are more difficult to achieve.

Dynamic mechanical analysis, DMA, is a non-destructive technique widely used in the characterization of the viscoelastic properties polymer-based systems including biomaterials covering wide temperature and frequency ranges [11]. Such studies are particularly relevant if the tests are performed at physiologically meaningful conditions, i.e., with the specimens completely incubated in solutions at $37^{\circ} \mathrm{C}$. Similar procedure was previously implemented to study starch-based biomaterials $[12,13]$. However, such dynamic tests performed at conditions close to the in vivo situation are not yet sufficiently established among the community. This work intends to strengthen the importance of such kinds of experiments.

In this study, the biodegradable polymeric scaffolds were prepared using compression molding technique with the melting of the polymer in a polymer/salt mixture followed by the dissolution of salt in water. One series of scaffolds was prepared by varying the polymer-to-salt ratio while keeping the salt size constant, and the other series of scaffolds was prepared by varying the sizes of the salt particles while keeping the polymer-to-salt ratio constant. The morphology of the scaffolds was assessed by X-ray microcomputed tomography analysis and scanning electron microscopy. The accessibility of internal pores or hydraulic conductivity of the scaffolds prepared with different pore sizes and porosities was determined by relative water uptake of the scaffolds. Moreover, the dynamic mechanical properties of the scaffolds were assessed in both dry and incubated state at physiological temperature of $37^{\circ} \mathrm{C}$. The relationship between mechanical properties, pore sizes and porosities were then established.

\section{Materials and methods}

\subsection{Materials and scaffold processing}

A 30/70 (wt.\%) blend of starch and PLLA (SPLA70) was used in this study. The SPLA70 was obtained by melt blending of $70 \mathrm{wt} . \%$ PLLA and $30 \mathrm{wt} . \%$ of corn starch. The PLLA of SPLA70 had L-lactide content of $94 \%$.

\subsubsection{Powder preparation and compounding}

SPLA70 pellets were dried at $50^{\circ} \mathrm{C}$ in a vacuum oven and cryogenically milled in an ultra-centrifugal mill (Retsch, ZM-100) with liquid nitrogen at $14,000 \mathrm{rpm}$. The selected $\mathrm{NaCl}$ particle sizes were hand-sieved with the stacked stainless steel sieves (ISO3310, Endecotts Ltd., England).

\subsubsection{Compression molding}

The compression-molded disks of around $5 \mathrm{~mm}$ thickness and $80 \mathrm{~mm}$ diameter were prepared on a steel mold using a Moore hydraulic press (UK), with 50 ton capacity. Prior to compression molding, the powdered SPLA70 was dried in a vacuum oven. The polymer/salt mixtures were put in between Teflon release papers in the mold. The mold was then placed in between the hot plates of the hydraulic press at top and bottom platen temperature of $180{ }^{\circ} \mathrm{C}$. To remove the trapped air in bulk powder, the pressure was slowly raised around $20 \mathrm{MPa}$ and released, and this process was repeated five times. The polymer/salt mixture was then allowed to melt for $6 \mathrm{~min}$ at constant pressure of $151 \mathrm{kPa}$, the weight of top half of the mold. The mold was then cooled to room temperature. The initial compositions of the constituents that were used to prepare the studied scaffolds are shown in Table 1.

\subsubsection{Porogen leaching}

The molded disks were sliced to $\sim 5 \times 5 \times 5 \mathrm{~mm}^{3}$ cuboids and put into water. The leaching was performed in beakers filled with de-ionized water at $37^{\circ} \mathrm{C}$. Water was replaced every $4 \mathrm{~h}$. The presence of $\mathrm{NaCl}$ content 
Table 1

Porous SPLA70 scaffolds prepared by compression molding of SPLA70 and $\mathrm{NaCl}$ mixtures followed by salt leaching

\begin{tabular}{lllll}
\hline Scaffolds & $\mathrm{NaCl}$ size $(\mu \mathrm{m})$ & \multicolumn{2}{l}{$\mathrm{NaCl}$ fraction } & Porosity \\
\cline { 3 - 5 } & & $(\mathrm{wt} . \%)$ & $(\mathrm{vol} . \%)$ & $(\%)$ \\
\hline $\mathrm{A} 1$ & $250-500$ & 70 & 59 & 73.9 \\
$\mathrm{~A} 2$ & $250-500$ & 80 & 70 & 78.6 \\
$\mathrm{~A} 3$ & $250-500$ & 85 & 78 & 82.0 \\
$\mathrm{~B} 1$ & $125-250$ & 80 & 70 & 80.2 \\
$\mathrm{~B} 2$ & $250-500$ & 80 & 70 & 78.6 \\
$\mathrm{~B} 3$ & $500-1000$ & 80 & 70 & 77.9 \\
\hline
\end{tabular}

$\mathrm{A} 2=\mathrm{B} 2$.

was checked by silver nitrate solution acidified with dilute nitric acid. The leaching protocol was continued till $48 \mathrm{~h}$.

The pyrolysis of the porous scaffolds was performed in a muffle furnace. The scaffolds were dried (in a vacuum oven for $24 \mathrm{~h}$ at $50{ }^{\circ} \mathrm{C}$ followed by cooling in a desiccator), weighed and placed in a crucible that was previously cleaned and dried. The crucible containing the scaffold was then placed inside the furnace. The temperature was raised slowly to $400{ }^{\circ} \mathrm{C}$ and kept for $5 \mathrm{~min}$, followed to $700{ }^{\circ} \mathrm{C}$ for $35 \mathrm{~min}$. Thereafter, the furnace was turned off, allowed to cool till $50{ }^{\circ} \mathrm{C}$. The crucible was taken out, kept in a desiccator for $24 \mathrm{~h}$ at room temperature and weighed. The wt.\% residue was calculated as

Residual salt content $=\left(\frac{m_{\mathrm{p}}}{m_{\mathrm{d}}}\right) \times 100$

where $m_{\mathrm{p}}$ and $m_{\mathrm{d}}$ are the weights of residue after pyrolysis and dried scaffold, respectively.

\subsection{Characterization of the scaffolds}

\subsubsection{Porosity and water uptake}

The porosities of the scaffolds were determined by X-ray microcomputed tomography (microCT) using a SkyScan1072 equipment (Belgium). The porous scaffolds were mounted on a rotary stage and scanned under a $40 \mathrm{kV}$ source for a complete rotation of $360^{\circ}$, during which 300 slices were produced. The resolution obtained was $8.79 \mu \mathrm{m} \mathrm{pixel}{ }^{-1}$.

The scaffolds were immersed in water and a series of short evacuation-repressurization cycles were carried out to force water into the pores of the scaffolds. The scaffolds were kept in water for $48 \mathrm{~h}$ to ensure the water impregnation into the open pores. The water uptake of the porous scaffolds was calculated as

water update $=\left(\frac{m_{\mathrm{w}}-m_{\mathrm{d}}}{m_{\mathrm{d}}}\right) \times 100$

where $m_{\mathrm{w}}$ is the weight of wet scaffold.

\subsubsection{Morphological characterization}

Porous morphologies of the scaffolds were examined using scanning electron microscopy (SEM) (Leica Cambridge $\mathrm{S}-360, \mathrm{UK}$ ) at $15 \mathrm{kV}$. The specimens were coated with gold using a sputter coater (JFC 1100, Jeol). The internal porous morphologies were characterized using microCT analysis.

\subsubsection{Dynamic mechanical analysis}

In dynamic mechanical analysis the specimens are subjected to a sinusoidal load, with a rate defined by a frequency $f$ (in cycles $\mathrm{s}^{-1}$ or $\mathrm{Hz}$ ) or an angular frequency $\omega=2 \pi f\left(\right.$ in $\left.\operatorname{rad~s}^{-1}\right): \sigma=\sigma_{0} \exp (\mathrm{i} \omega t+\delta)$, where $\mathrm{i}=(-1)^{1 / 2}$. The response (a strain) though sinusoidal is not in phase with the developed stress, and lags behind the stress by some phase angle $\delta$ between 0 and $90^{\circ}$. In this case, the modulus has a complex nature:

$$
\begin{aligned}
E^{*}(\omega) & =\frac{\delta}{\epsilon}=\left(\sigma_{0} / \epsilon_{0}\right) \exp (\mathrm{i} \delta)=\left(\delta_{0} / \epsilon_{0}\right)(\cos \delta+\mathrm{i} \sin \delta) \\
& =E^{\prime}+i E^{\prime \prime}
\end{aligned}
$$

The storage modulus, $E^{\prime}$, is the elastic or real component of complex modulus, $E^{*}$, and is in phase with $\sigma$. The storage modulus is related to the amount of energy stored by the material during a cycle. The loss modulus, $E^{\prime \prime}$, is the viscous component of $E^{*}$, and is $\pi / 2$ out of phase with the stress, and is associated with the dissipation of energy due to internal friction at the microscopic level. The loss factor, $\tan \delta=E^{\prime \prime} / E^{\prime}$, is a measure of the damping capability of a material.

The dynamic mechanical analysis (DMA) was performed on the porous three-dimensional scaffolds under compression in a TRITEC 2000B DMA (Triton Technology, UK). The dry state measurements of the porous scaffolds with dimensions around $5 \times 5 \times 5 \mathrm{~mm}^{3}$ were carried out at a physiological temperature of $37^{\circ} \mathrm{C}$. The tests were performed under compression from 0.05 to $50 \mathrm{~Hz}$ at a constant dynamic displacement of $50 \mu \mathrm{m}$. Five points were measured within each decade.

For the measurements at the wet state, a phosphate buffer solution (PBS) was forced into the scaffolds by brief evacuation-repressurization cycles and kept in a soaked condition for $45 \mathrm{~min}$ prior to placing it into PBS bath for the DMA experiments. The measurements were carried out under compression by immersing the wet scaffolds in PBS bath at a constant physiological temperature of $37^{\circ} \mathrm{C}$. The temperature was equilibrated by keeping the scaffolds $15 \mathrm{~min}$ in the PBS bath of DMA. The tests were performed under compression in the frequency range of $0.05-10 \mathrm{~Hz}$ at a constant dynamic displacement of $50 \mu \mathrm{m}$. For each individual scaffold, the data is averaged over three consecutive runs. Three samples were measured for each type of scaffolds.

\section{Results and discussion}

\subsection{Scaffold characterization}

The three-dimensional porous scaffolds can be conveniently prepared by compression molding of thermoplastic 
polymer powder and salt particulate mixtures followed by the removal of salt fraction. In the compression molding technique, the sintering of bulk polymer/salt mixture into a homogeneous compact form through melting depends on the viscosity of the molten polymer. The melting temperature of this PLLA in SPLA70 was $\sim 155^{\circ} \mathrm{C}$ [14]. However, the porous three-dimensional scaffolds prepared in the temperature range of $155-170{ }^{\circ} \mathrm{C}$ were not homogeneous due to limited fluidity of the molten polymer. The viscosity of PLLA is sensitive to temperature and decreases rapidly with increasing temperature [15]. Moreover, both PLA and starch are sensitive to degradation at elevated temperature. The molding temperature of $180^{\circ} \mathrm{C}$ and residence time of 6 min are a compromise between degradation and fluidity of PLLA that allowed producing homogeneous scaffold with minimal degradation of the constituent polymers.

With the addition of different fraction of salt particulates, the scaffold porosity can be tuned. In this case, the concern arises with the minimum and maximum fractions of salt that can be added to obtain stable scaffolds with minimum or no salt content. Typically, the maximum packing of mono-dispersed dense spheres is $68 \mathrm{vol} \%$. In the present study, starting from $60 \mathrm{wt} . \%$ with a step of $10 \mathrm{wt} . \%$, the maximum salt concentration of $90 \%$ was tested. Below 70 wt. $\%$ of salt fraction, the salt particles cannot be fully extracted. The porous scaffolds prepared with salt fraction of $90 \mathrm{wt} . \%$ are prone to disintegration. The stable scaffolds were achieved with $85 \mathrm{wt} . \%$ of pre-seeded salt particulate. The fabrication procedure for all the scaffolds were maintained the same.

One series of scaffolds was prepared with increasing porogen fraction while maintaining the constant pre-seeded salt size (Table 1). Fig. 1 shows that the distribution of pores was uniform throughout the matrix. The microCT images in Fig. 1 show that the number of pores is increasing from left (A1) to right (A3), as expected. The second series of scaffolds are prepared with varying pore sizes while the porogen fraction was kept constant. As expected, Fig. 1 shows that the pore sizes are increasing from top (B1) to bottom (B3) with increasing porogen sizes.

The SEM micrograph in Fig. 2 shows the pore size and pore wall distribution on a higher magnification. The number of pores is increasing from left to right, i.e. with increasing porogen fraction. However, with the higher fraction of salts, there are some pores larger than the size of salts. This is expected as salt-salt contact points increases with more than 68 vol.\% pre-seeded particles; in such cases some of the resulting agglomerates will give rise, upon leaching, to pore sizes bigger than the pre-seeded salt size.

It is clearly visible in the higher-magnification SEM micrographs in Fig. 2 that the pore sizes are increasing from top to bottom, i.e. with increasing pre-seeded salt size at constant fraction of salt. It has been frequently observed

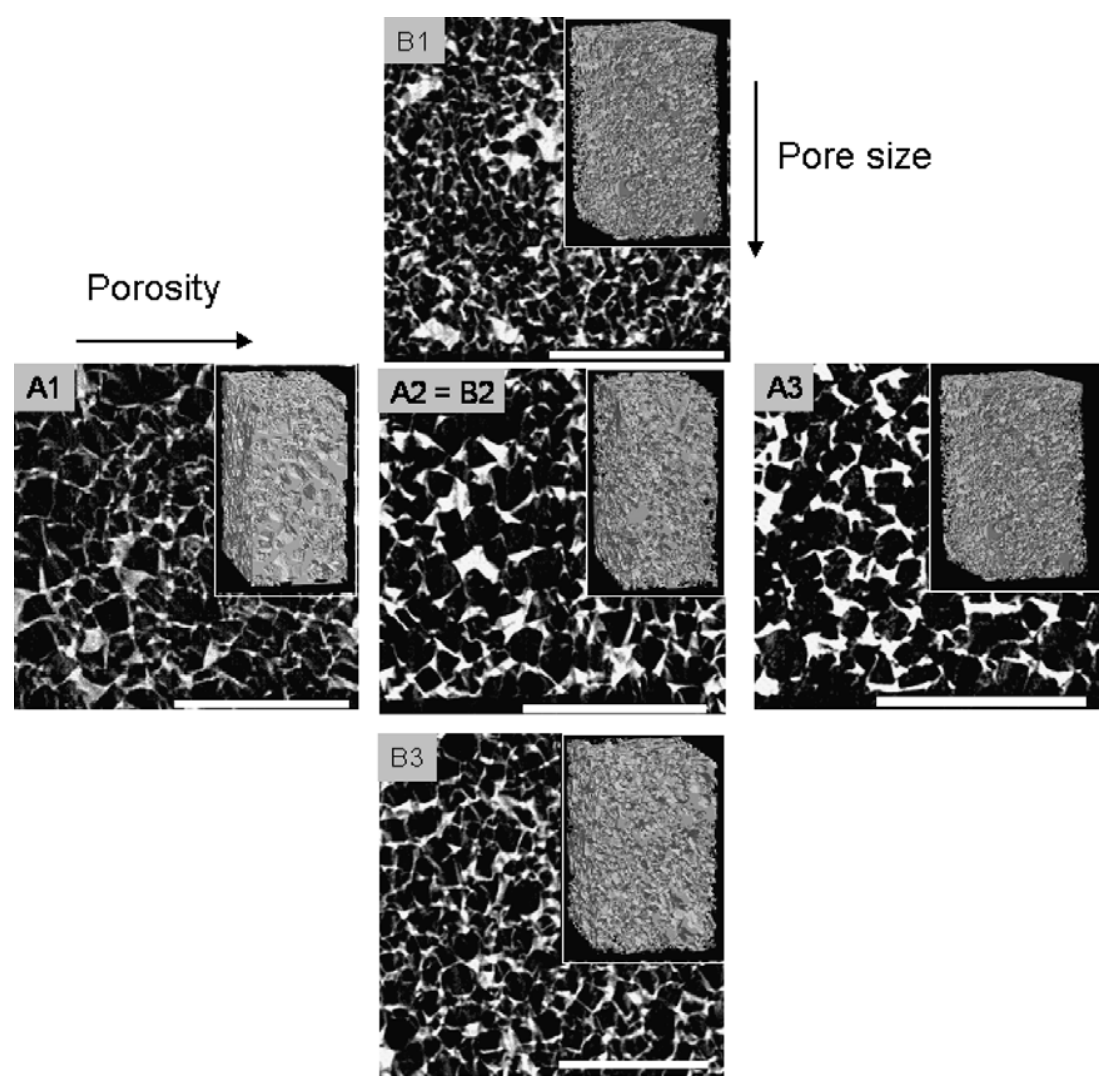

Fig. 1. Representative two-dimensional microCT images showing the homogeneous pore distribution across the porous scaffolds, the insets represents the microCT reconstruction of three-dimensional scaffolds. The scale bar $=2 \mathrm{~mm}$. 


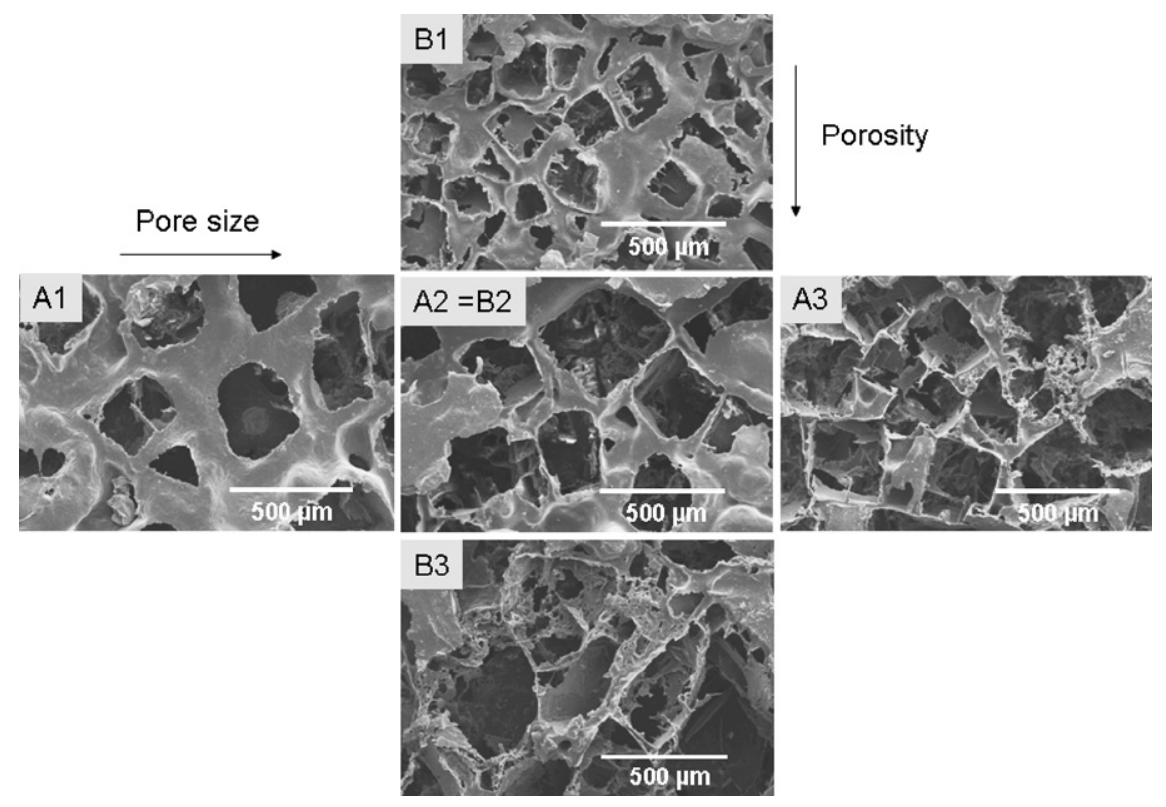

Fig. 2. Representative SEM micrographs of the scaffolds showing the increase of porosities along the horizontal direction prepared with constant salt size of $250-500 \mu \mathrm{m}$ and increase of pore sizes along the vertical direction prepared with constant salt fraction of 80 wt. $\%$.

with big salt sizes, that a number of small pores $(5-10 \mu \mathrm{m})$ appear on the pore wall and the pore wall thickness was non-uniform. The smaller pores in the pore walls may offer advantages in tissue engineering application, as it increases the interconnectivity.

Fig. 3 shows that the apparent porosities were increased for all the scaffolds with respect to the pre-seeded salt fraction. The difference between apparent porosities and preseeded salt fractions were decreased with increasing salt fraction. The possible reason for the higher apparent porosity compared with pre-seeded salt fraction could be the loss of poorly integrated polymeric fragments during leaching of porogen. Similar results were obtained with PLLA, SPLA50 (blend of 50:50 wt.\% starch and PLLA), composites of PLLA/hydroxyapatite porous composites prepared with compression molding and salt leaching technique $[16,17]$.

An increase in apparent porosities was also observed with scaffolds prepared with increasing salt size at constant fraction of pre-seeded salt particulates. Surprisingly, the results show that the apparent porosities are decreasing with increasing pre-seeded salt size. Similar results were also reported with poly(ether ester) copolymer scaffolds prepared with compression molding and salt leaching technique [18]. Nevertheless, the variation of porosity when different salt particle sizes are used is small $(77.9-80.2 \%$ in the extremes), when compared with the changes in pore sizes. Therefore we can consider that any change observed in this set of scaffolds, B1, B2 and B3, will be mainly a consequence of the pore sizes.

Fig. 4 shows the porosity distribution across the scaffolds obtained from the analysis of the microCT results. The porosity distribution is quite uniform throughout the scaffold. The distribution is narrower with both the increased pre-seeded salt sizes and salt fractions. The porosity distribution is broader with lowest fraction (70 wt.\%) of pre-seeded salt fraction (A1).

The porous scaffolds prepared with $70-85 \mathrm{wt} . \%$ of preseeded $\mathrm{NaCl}$ particulates do not contain residual salt particles after $\mathrm{NaCl}$ leaching in water. This has been verified from the pyrolysis of the porous scaffolds. This result indicates that compression molding and salt leaching technique allows preparing salt-free and stable three-dimensional starch-based polymeric scaffolds with $74-82 \%$ porosity.

Fig. 5 shows that the SPLA70 scaffolds with the same pore size take up $217-366 \%$ of water (a variation of $69 \%$ ) with increasing porosities. The average hydraulic conductivities of porous SPLA70 scaffolds are higher than in PLLA scaffolds of similar porosities [16]. This could be due to the presence of hydrophilic starch that assists to uptake more water than the hydrophobic PLLA fraction, contributing to an increase of the total water uptake for SPLA70 scaffolds. As expected, the water uptake follows the same trend of porosities for A1, A2 and A3 scaffolds. The corresponding porosities of A1 and A3 were 73.9 and $82 \%$, respectively (a variation of $11 \%$ ). So, the increment in water uptake is substantially higher than the increment in porosity. The hydraulic conductivity increases significantly with the increasing of porosities from $73.9 \%$ to $82 \%$. The higher water uptake compared with the increment of porosity has been reported with other polymeric scaffolds prepared with compression molding and salt leaching technique [19].

For B1, B2 and B3 scaffolds, the water uptake increases with increasing pore size. The average water uptake of B3 scaffolds (pore size $500-1000 \mu \mathrm{m}$ ) was $380 \%$, and B1 scaffolds (pore size 125-250 $\mu \mathrm{m}$ ) had 239\% (a variation of $59 \%$ ). Note that the B3 scaffolds had slightly lower porosity 


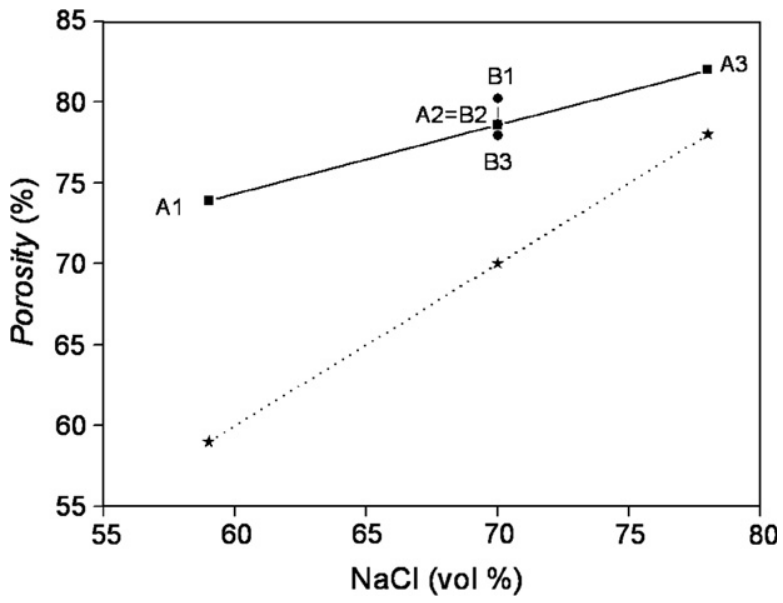

Fig. 3. Comparison between the apparent porosities obtained with respect to the fraction and size of the pre-seeded salt particles. The dotted line represents the theoretical porosity.
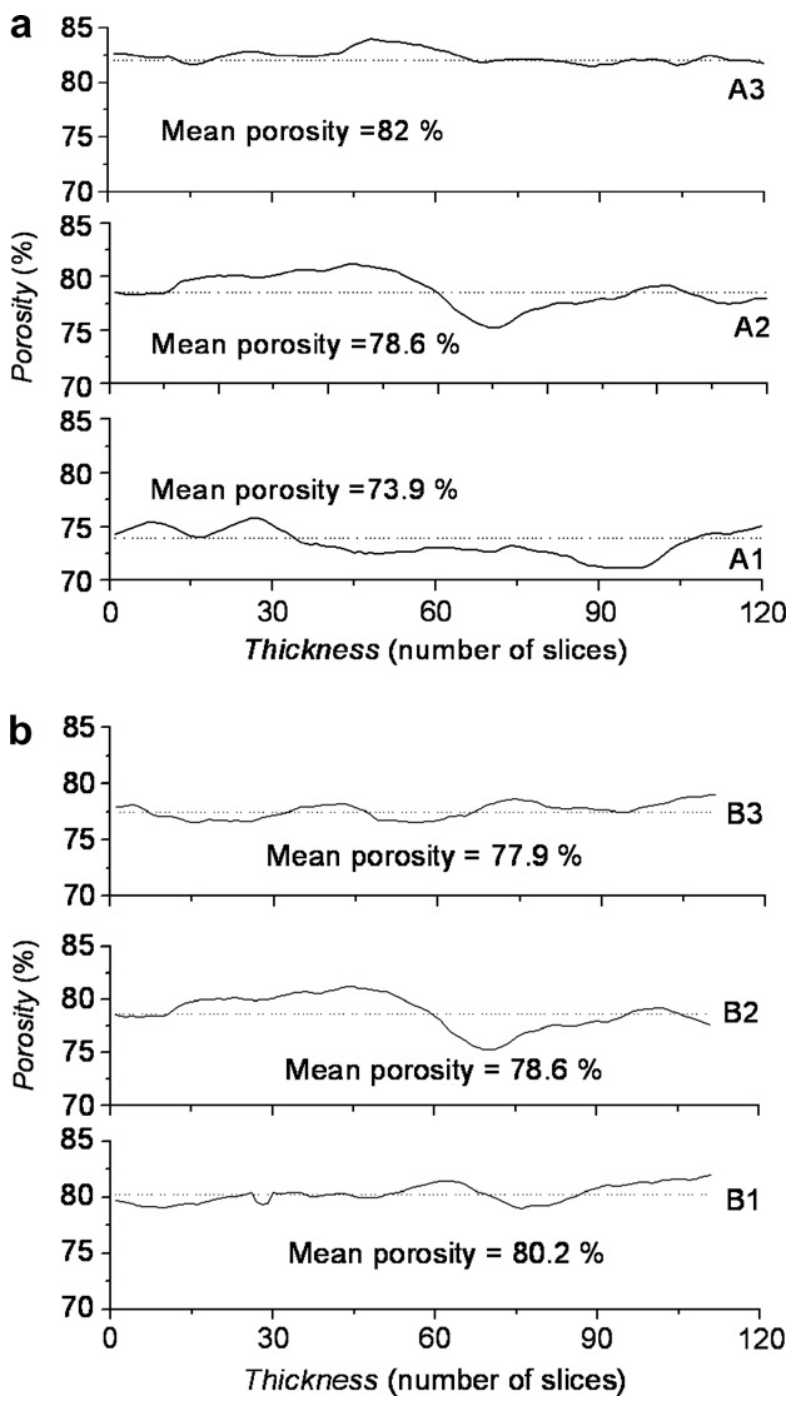

Fig. 4. Representative porosity distribution along the thickness of the porous scaffolds: (a) effect of pre-seeded salt fractions at constant salt size of $250-500 \mu \mathrm{m}$, and (b) varying salt sizes at constant salt fraction of 80 wt. $\%$.

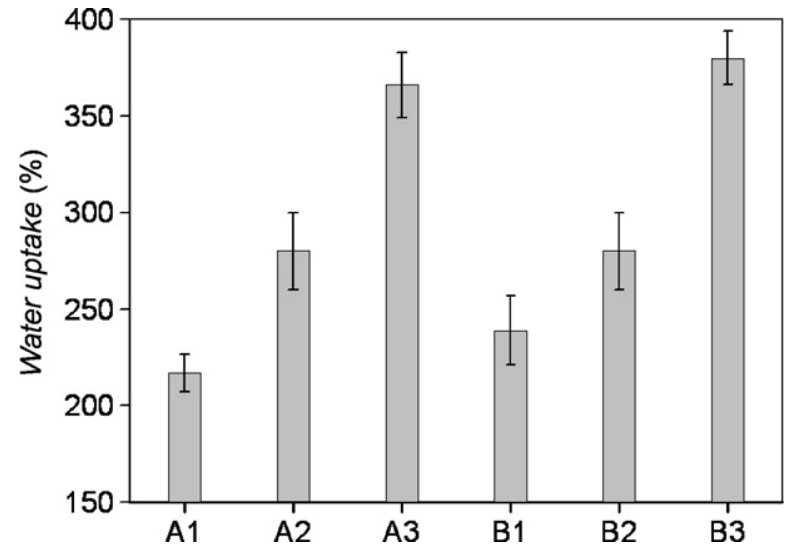

Fig. 5. Variations of equilibrium water uptake with the variation of porosity and pore size of SPLA70 scaffolds at $37^{\circ} \mathrm{C}$ and at atmospheric pressure.

than B1 scaffolds (a variation of $\sim 3 \%$ ). The average salt particle sizes of B1 scaffolds were nearly four times less than the corresponding salt particle sizes of B3. Therefore, the theoretical pore surface area of B1 scaffolds is around four times higher than B3 scaffolds. If the pore surface area is accountable for the water uptake of the scaffolds, it should follow the order $\mathrm{B} 1>\mathrm{B} 2>\mathrm{B} 3$. However, the water uptake of the scaffolds follows B3 $>$ B2 $>$ B1 (see Fig. 5). The reason for the discrepancy is likely due to trapped air in the bulk pores, which could be increased with increasing surface areas of decreasing pore sizes. These voids may not allow water to permeate into the pores, thus results a lower uptake with decreasing pore size. Moreover, it could be clearly evident from the micrographs of B2 and B3 in Fig. 2 that the number of small pores in the walls separating the bigger pores created by dissolution of salt particles are increasing with increasing salt sizes. This perforation in pore walls may have facilitated the hydraulic conductivity that led to the higher water uptake of the scaffolds with bigger pores.

\subsection{Dynamic mechanical properties}

The solid-state rheological properties of the starchbased scaffolds with different porosities and pore sizes were determined by DMA. The storage modulus and the loss factor of these two sets of scaffolds are shown as a function of frequency in Fig. 6, where the measurements were performed in dry state. No evident variations along the frequency axis are seen in these curves, indicating that the viscoelastic properties of the scaffolds are quite steady within a large time scale range; this suggests that no discrete relaxation process takes place in this frequency window at $37^{\circ} \mathrm{C}$. The possible reason for the steady viscoelastic performance of the scaffolds could be due to the fact that the material was studied at $37^{\circ} \mathrm{C}$, and its glass transition temperature is about $60^{\circ} \mathrm{C}$ [14].

The values found in Fig. 6 for a frequency of $1 \mathrm{~Hz}$ are shown in Fig. 7, as a function of porosity. The results vary 

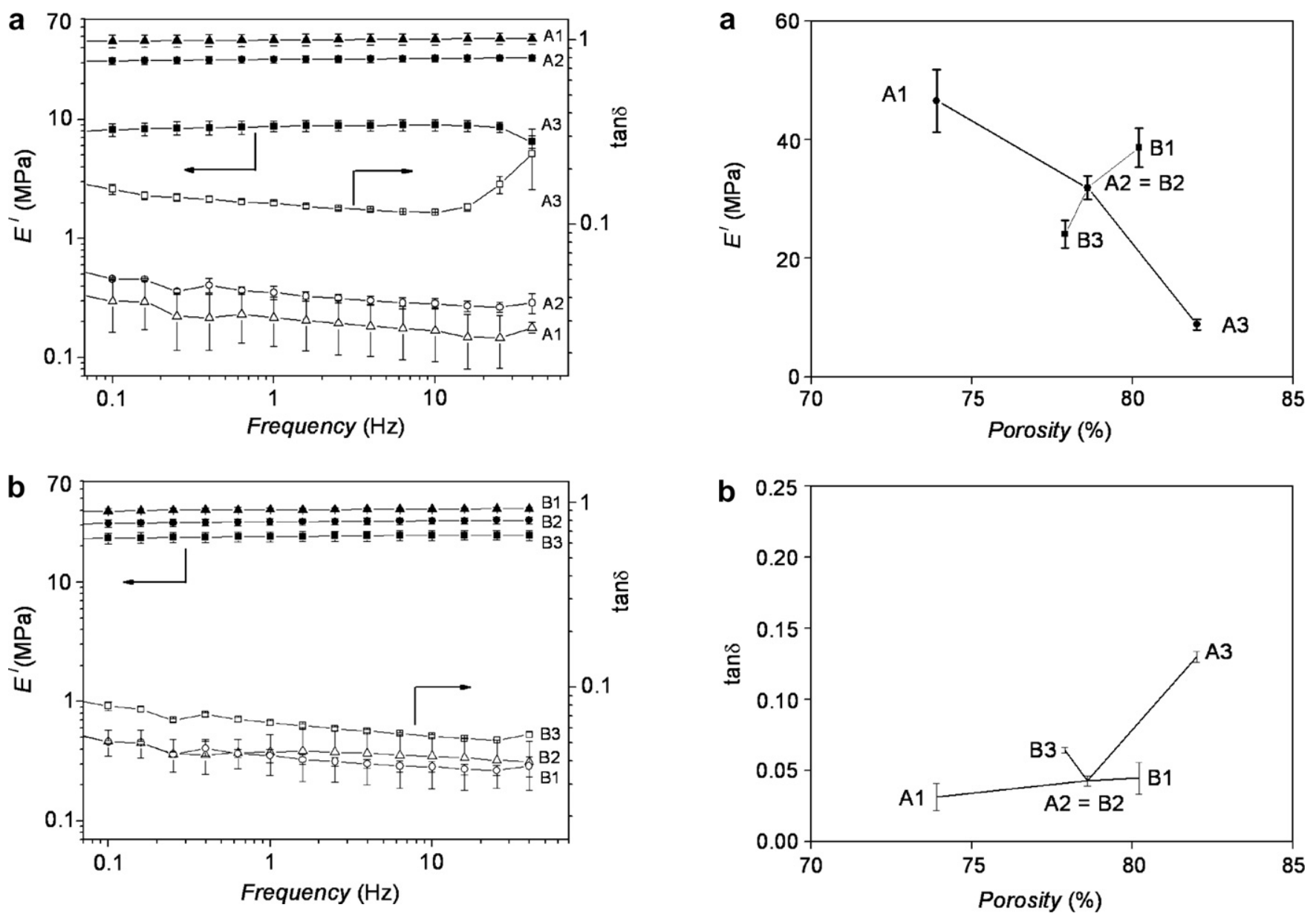

Fig. 6. Variation of $E^{\prime}$ (filled symbols) and $\tan \delta$ (open symbols) with frequency for the different porous SPLA70 scaffolds. The experiments were carried out at $37^{\circ} \mathrm{C}$ and at dry state. (a) Effect of porosity on the scaffolds prepared with constant salt size of $250-500 \mu \mathrm{m} \quad(\mathrm{A} 1=\boldsymbol{\Lambda}$, $\mathrm{A} 2=\boldsymbol{\bullet}, \mathrm{A} 3=\mathbf{\square})$; (b) effect of pore size on the scaffolds with constant salt fraction of 80 wt. $\%(B 1=\boldsymbol{\Lambda}, \mathrm{B} 2=\boldsymbol{\bullet}, \mathrm{B} 3=\mathbf{\square})$.

from $5 \mathrm{MPa}$ to $45 \mathrm{MPa}$, depending on the structure. For comparison, the flexural storage modulus at $37^{\circ} \mathrm{C}$ measured at $1 \mathrm{~Hz}$ for compact SPLA70 was found to be $1400 \mathrm{MPa}$ [12], i.e. around two orders of magnitude higher than the scaffolds. As expected, the storage modulus decreases with increasing porosity for the group A1, A2, A3 (Fig. 7a). However, the results from the group B1, B2, B3 also allow us to conclude that the pore size plays an important role in the stiffness of the scaffolds: for nearly the same porosities, the storage modulus decreases with increasing pore size. The similar trend was observed with scaffolds prepared with salt leaching techniques but with other materials [18]. The values of the loss factor at $f=1 \mathrm{~Hz}$ in Fig. 7b indicates that in general most of the values do not correlate with either porosity or with pore size. Using a basic viscoelastic model considering a porous system as a combination in parallel of a solid material, corresponding to the matrix, coupled with an empty space, corresponding to the pores, this should be expected, where the loss factor of the ensemble is the one of the matrix. In

Fig. 7. (a) Variation of $E^{\prime}$ with porosity (-) and pore size ( $\left.\mathbf{\square}\right)$ of the SPLA70 scaffolds at $1 \mathrm{~Hz}$. (b) Variation of $\tan \delta$ with porosity (-) and pore size (ם) of the SPLA70 scaffolds at $1 \mathrm{~Hz}$. All the experiments were carried out at $37^{\circ} \mathrm{C}$ and at dry state.

fact, the loss factor of the compact material was found to be 0.028 , at $37^{\circ} \mathrm{C}$ and $1 \mathrm{~Hz}$ [12], which is similar to most of the values in Fig. 7b. However, it is clear that, for A3, the $\tan \delta$ value is significantly higher compared with other scaffolds; this also happens in the entire frequency range explored (see Fig. 6). This behavior should be explained by other dissipation mechanisms that occur preferentially in such specimens with higher porosity.

One possibility could be a friction effect of the perforation of the pore walls, frequently observed in higher porosity scaffolds of A3 (Fig. 2) compared with compact surfaces of A1 (Fig. 2). It would be also interesting to compare the obtained values of $\tan \delta$ with those found in tissues. For example, Buechner et al. [20] collected a series of results of $\tan \delta$ of bone in a frequency range of $10^{-7}-10^{7} \mathrm{~Hz}$. The values of the loss factor ranged between 0.01 and 0.1 , but for frequencies physiologically felt by living bone, say $10^{-2}-10^{3} \mathrm{~Hz}$, the values for human bone varies typically between 0.02 and 0.04 . This interval is in agreement with the order of magnitude of the values of $\tan \delta$ found in the studied scaffolds, not only at $1 \mathrm{~Hz}$ but in all frequency ranges explored (see Fig. 6). 
As the modulus of a porous scaffold is related to the modulus of parent material and porosity, it is reasonable to compare the normalized modulus, i.e. the ratio of the modulus of the porous scaffold with the modulus of compact material, with normalized density, i.e. the ratio of the apparent density of the porous scaffold with the density of compact material. For porous construct, the relationship between normalized modulus and normalized density is related by the following expression [21]:

$\frac{E}{E_{c}}=C\left(\frac{\rho}{\rho_{c}}\right)^{n}$

where $E$ and $E_{c}$ are the moduli of porous scaffold and solid material, respectively; $\rho$ and $\rho_{\mathrm{c}}$ are the densities of porous scaffold and solid material, respectively. The constants $C$ and $n$ depend on the microstructure of the scaffold. The constant $C$ includes all geometric constants of proportionality. The value of $n$ generally lies in the range of $1<n<3$, resulting a wide range of values for modulus at a given density [18]. For an isotropic open cell structure, the exponent $n$ is expected to be 2. A number of factors affect modulusporosity relationship, such as open and closed pores; the distribution and dispersion of pores; and the shape and orientation of pores. The dependence of $\mathrm{C}$ and $n$ on the microstructure of highly porous scaffolds with random distribution of pores, such as the ones investigated in the present study, is not well understood.

The values of $n$ were calculated from Eq. (4) by putting the experimental values of moduli for all the SPLA70 scaffolds at $1 \mathrm{~Hz}$, the modulus of compact SPLA70 as $1400 \mathrm{MPa}$ [12] and $C=1$. The $n$ values for $\mathrm{A} 1, \mathrm{~A} 2$ and $\mathrm{A} 3$ scaffolds were 2.53 , 2.45 and 2.96, respectively; and for B1, B2 and B3 scaffolds were $2.22,2.45$ and 2.69 , respectively. Thus the values of $n$ increase with increasing porosities and also with increasing pore sizes. The experimental data fits reasonably well with the theoretical model with the exponent values $2<n<3$ with $C=1$, and is appropriate to describe the elastic modulus of porous scaffolds $[18,22]$. Such an approach could enable scaffolds to be designed with a given stiffness adequate for a desired tissue engineering application.

DMA experiments were also performed on samples in immersed state, in order to estimate the viscoelastic properties of the specimens in more realistic physiological conditions. The results obtained at $37^{\circ} \mathrm{C}$ are shown in Fig. 8, where the two sets of samples are shown separately. The viscoelastic properties are quite steady along the frequency range analyzed, although the $\tan \delta$ values in immersed state tend to decrease more with increasing frequency compared with the dry state measurement. Especially for the samples with higher porosity, it is found that the storage modulus is similar to the dry samples. This is somewhat unexpected as the material can take up some quantity of water and the stiffness is expected to decrease upon immersion. However, in the case of immersed samples the pores are filled with water that could render more difficult the deformability of the scaffold through a hydrostatic effect.
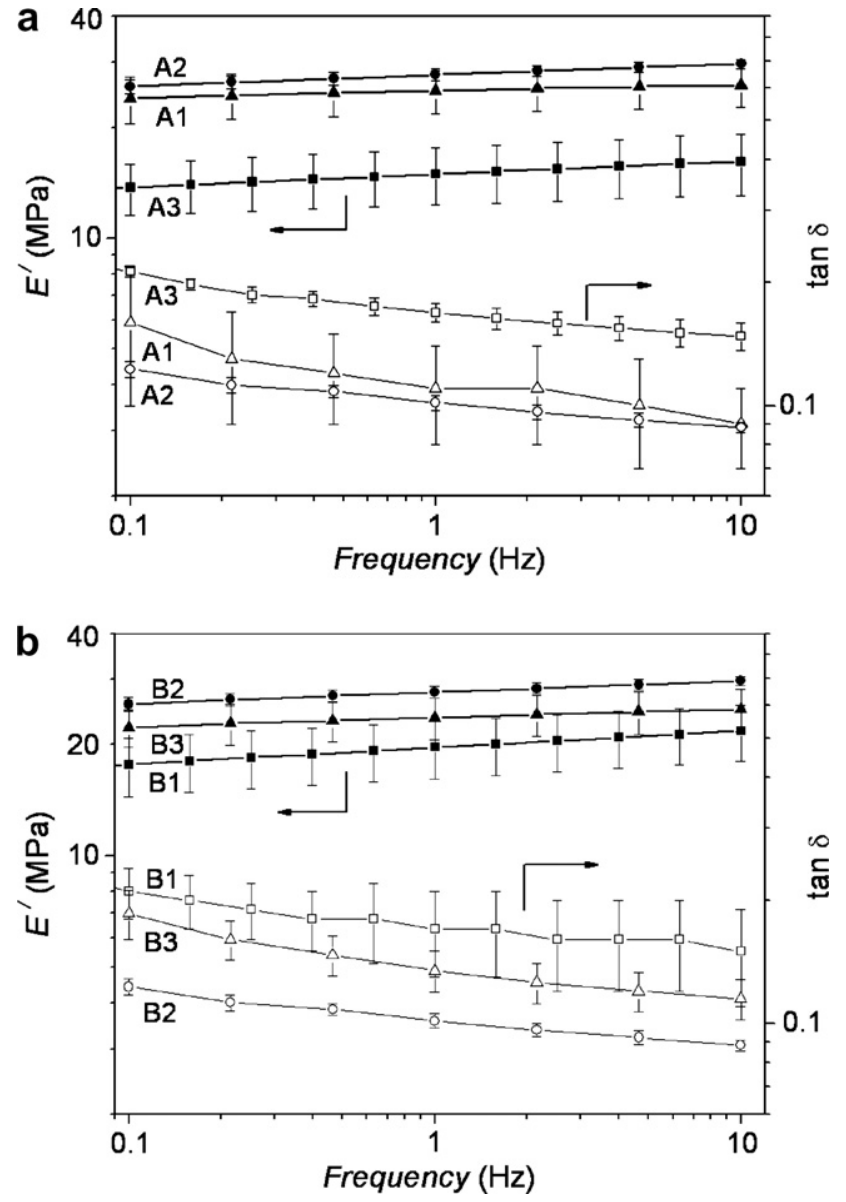

Fig. 8. Variation of $E^{\prime}$ (filled symbols) and $\tan \delta$ (open symbols) with frequency for the different porous SPLA 70 scaffolds at $37^{\circ} \mathrm{C}$, measured in PBS. The experiments were carried out at $37^{\circ} \mathrm{C}$ and in immersed state. (a) Effect of porosity on the scaffolds prepared with constant salt size of 250 $500 \mu \mathrm{m}$. (A1 $=\boldsymbol{\Lambda}, \mathrm{A} 2=\boldsymbol{\bullet}, \mathrm{A} 3=\mathbf{\square})$; (b) Effect of pore size on the scaffolds with constant salt fraction of 80 wt. $\%(B 1=\boldsymbol{\Lambda}, \mathrm{B} 2=\boldsymbol{\bullet}, \mathrm{B} 3=\mathbf{\square})$.

The values of $\tan \delta$ for the wet samples are higher than for the dry samples (Fig. 6). The contribution of the friction between the water molecules and the construct could be responsible for this increment in the loss factor.

The results at $1 \mathrm{~Hz}$ extracted from Fig. 8 are shown in Fig. 9 as a function of porosity. The effect on the scaffolds' stiffness of this parameter, as well as the effect of the pore size, is almost lost, as compared with the data obtained for the dry samples. Such behavior strengthens the hypothesis of the hydrostatic effect of the water within the pores that makes difficult the deformability of the construct. There is also no apparent effect of porosity and pore size on the loss factor (Fig. 9b).

The mechanical properties of scaffolds are widely reported in the literature, but most of the tests are performed in dry samples. In this work it is clear that the viscoelastic properties of the studied scaffolds are different when the samples are measured under physiological conditions. Therefore, any comparison of the mechanical performance of polymeric constructs with biomechanical properties of tissues should ideally made with experimental 

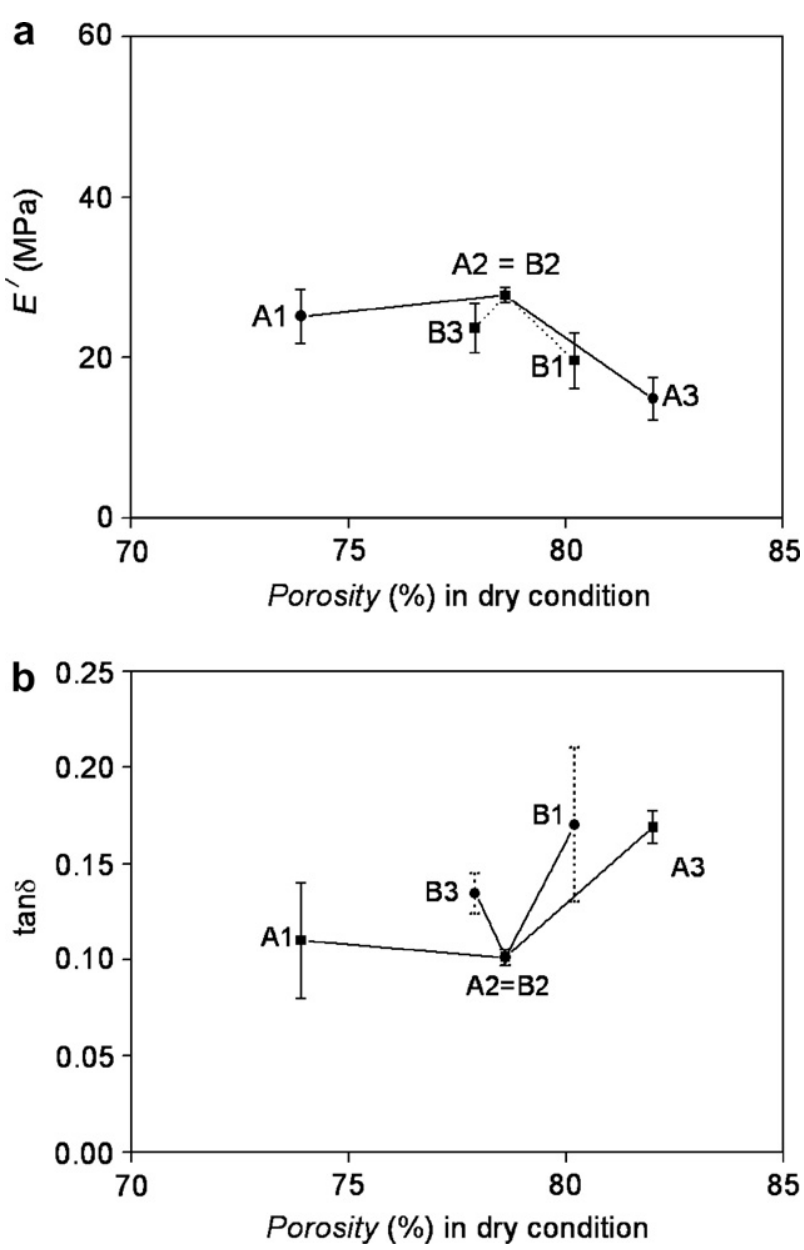

Fig. 9. (a) Variation of $E^{\prime}$ with porosity (0) and pore size (ם) of the SPLA70 scaffolds at $1 \mathrm{~Hz}$. (b) Variation of $\tan \delta$ with porosity (-) and pore size $(\square)$ of the SPLA70 scaffolds at $1 \mathrm{~Hz}$. The experiment was carried out at $37^{\circ} \mathrm{C}$ and in immersed state.

data taken on immersed samples in physiological simulated solutions, at $37^{\circ} \mathrm{C}$. We believe that it is not enough to wet the samples and measure them in conventional equipments; for the case of scaffolds, the samples should be completely immersed during the measurements.

\section{Conclusions}

Three-dimensional scaffolds of a blend of starch and poly(L-lactic) acid), SPLA70, were produced by compression molding followed by leaching of salt and characterized in terms of morphology, water uptake and viscoelastic properties using dynamic mechanical analysis. The results indicate that scaffolds with porosities of $74-82 \%$ can be prepared with the procedure employed. The structures exhibited homogeneous porosities throughout the entire volume. The hydraulic conductivity of the porous SPLA70 scaffolds increases with increasing pre-seeded porogen size, as indicated with water uptake capability.

As expected, the elastic moduli of the dry scaffolds have been decreased with increasing porosity. However, pore size also plays an important role in the mechanical perfor- mance of the scaffolds. Apparently, the morphology did not influence strongly the damping properties of the scaffolds; however, for the highest porosities the loss factor was higher than in the other cases that exhibit values close to human bone.

In situ DMA experiments were also performed with the samples immersed in a physiological simulated solution of $37^{\circ} \mathrm{C}$. The viscoelastic and mechanical performance of such scaffolds measured in these conditions are significantly different compared with dry state measurement. In immersed state, the stiffness was much less dependent on the pore architecture and porosity, probably due to the hydrostatic effect of water entrapped within the pores that could also explain the higher values of the loss factor in the immersed samples.

From the obtained results it can be concluded that DMA is a suitable technique to assess the solid-state rheological properties of scaffolds for tissue engineering applications allowing for measurements in simulated physiological conditions of hydration and temperature.

\section{Acknowledgements}

Financial support for this work was provided by FCT, through the POCTI and FEDER programmes (POCTI/ FIS/61621/2004) and the European Union funded STREP Project HIPPOCRATES (NMP3-CT-2003-505758). S. Ghosh thanks the Portuguese Foundation for Science and Technology (FCT), Government of Portugal, for awarding the PhD Grant (SFRH/BD/12657/2003).

\section{References}

[1] Murphy WL, Kohn DH, Mooney DJ. Growth of continuous bonelike mineral within porous poly(lactide-co-glycolide) scaffolds in vitro. J Biomed Mater Res 2000;50:50-8.

[2] Chupa JM, Foster AM, Sumner SR, Madihally SV, Matthew HWT. Vascular cell responses to polysaccharide materials: in vitro and in vivo evaluations. Biomaterials 2000;21:2315-22.

[3] Suzuki M, Ikada Y. Biodegradable polymers in medicine. In: Reis RL, San Román J, editors. Biodegradable systems in tissue engineering and regenerative medicine. NY: CRC Press; 2005. p. 3-12.

[4] Middleton JC, Tipton AJ. Synthetic biodegradable polymers as orthopedic devices. Biomaterials 2000;21:2335-46.

[5] Mano JF et al. Natural origin biodegradable systems in tissue engineering and regenerative medicine: present status and some moving trends. J Roy Soc Interface 2007;4:999-1030.

[6] Athanasiou KA, Agrawal CM, Barber FA, Burkhart SS. Orthopaedic applications for PLA-PGA biodegradable polymers. Arthroscopy 1998;14:726-37.

[7] Marques AP, Cruz HR, Coutinho OP, Reis RL. Effect of starchbased biomaterials on the in vitro proliferation and viability of osteoblast-like cells. J Mater Sci - Mater Med 2005;16:833-42.

[8] Harris LD, Kim BS, Mooney DJ. Open pore biodegradable matrices formed with gas foaming. J Biomed Mater Res 1998;42:396-402.

[9] Mikos AG, Thorsen AJ, Czerwonka LA, Bao Y, Langer R, Winslow $\mathrm{DN}$, et al. Preparation and characterization of poly(L-lactic acid) foams. Polymer 1994;35:1068-77.

[10] Moroni L, De Wijn JR, Van Blitterswijk CA. 3D fiber-deposited scaffolds for tissue engineering: influence of pores geometry and architecture on dynamic mechanical properties. Biomaterials 2006;27:974-85. 
[11] Mano JF, Reis RL, Cunha AM. Dynamic mechanical analysis in polymers for medical applications. In: Reis RL, Cohn D, editors. Polymer based systems on tissue engineering, replacement and regeneration, Nato science series, Dordrecht. The Netherlands: Kluwer; 2002. p. 139-64.

[12] Mano JF, Reis RL. Viscoelastic monitoring of starch-based biomaterials in simulated physiological conditions. Mater Sci Eng A 2004;370:321-5.

[13] Alves NM, Saiz-Arroyo C, Rodriguez-Perez MA, Reis RL, Mano JF. Microhardness of starch based biomaterials in simulated physiological conditions. Acta Biomater 2007;3:69-76.

[14] Mano JF, Koniarova D, Reis RL. Thermal properties of thermoplastic starch/synthetic polymer blends with potential biomedical applicability. J Mater Sci - Mater Med 2003;14:127-35.

[15] Ghosh S, Viana JC, Reis RL, Mano JF. Effect of processing conditions on morphology and mechanical properties of injectionmolded poly(L-lactic acid). Polym Eng Sci 2007;47:1141-7.

[16] Ghosh S, Viana JC, Reis RL, Mano JF. The double porogen approach as a new technique for the fabrication of interconnected
poly(L-lactic acid) and starch based biodegradable scaffolds. J Mater Sci - Mater Med 2007;18:185-93.

[17] Ghosh S, Viana JC, Reis RL, Mano JF. Bi-layered constructs based on poly(L-lactic acid) and starch for tissue engineering of osteochondral defects. Mater Sci Eng C 2008;28:80-6.

[18] Hou Q, Grijpma DW, Feijen J. Porous polymeric structures for tissue engineering prepared by a coagulation, compression moulding and salt leaching technique. Biomaterials 2003;24:1937-47.

[19] Katoh K, Tanabe T, Yamauchi K. Novel approach to fabricate keratin sponge scaffolds with controlled pore size and porosity. Biomaterials 2004;25:4255-62.

[20] Buechner PM, Lakes RS, Swan C, Brand RA. A broadband viscoelastic spectroscopic study of bovine bone: implications for fluid flow. Ann Biomed Eng 2001;29:719-28.

[21] Gibson LJ. Biomechanics of cellular solids. J Biomech 2005;38:377-9.

[22] Blaker JJ, Maquet V, Jerome R, Boccaccini AR, Nazhat SN. Mechanical properties of highly porous PDLLA/Bioglass ${ }^{\circledR}$ composite foams as scaffolds for bone tissue engineering. Acta Biomater. 2005;1:643-52. 